\title{
Loss of tumor intrinsic PD-L1 confers resistance to drug-induced apoptosis in human colon cancer
}

\author{
Daofu FENG ${ }^{1}$, Zuoyu $\mathrm{CHEN}^{1}$, Xianghui $\mathrm{HE}^{1}$, Shengbing HUANG², Zhixiang ZHANG ${ }^{1, *}$ \\ ${ }^{1}$ Department of General Surgery, Tianjin Medical University General Hospital, Tianjin, China; ${ }^{2}$ Department of Biomedical Informatics, Mayo \\ Clinic, Rochester, MN, USA \\ *Correspondence: zhangzhixiang63@sina.com
}

Received May 31, 2020 / Accepted August 21, 2020

\begin{abstract}
Colorectal cancer (CRC) with BRAF (V600E) is associated with microsatellite instability (MSI) that predicts response to immune checkpoint inhibitors. We demonstrated the interrogation of TCGA RNA-seq human datasets revealed that BRAFV600E tumors had significantly higher Programmed Death Ligand 1 (PD-L1) mRNA compared to non-mutated BRAF CRCs. Also, MSI-H tumors were evaluated as higher PD-L1 than MSS CRCs. Inhibition of MEK/ERK by cobimetinib or CDK inhibitor dinaciclib was shown to attenuate mutant BRAF-induced PD-L1 coincident with reduced c-JUN and YAP expression whose combined knockdown reduced PD-L1. Using TCGA datasets, PD-L1 mRNA expression in human colon cancers was significantly associated with YAP expression. The deletion of PD-L1 can reduce tumor cell growth shown by clonogenic assay. Analysis of the role of PD-L1 as a mediator of chemosensitivity was then performed. Knockout of PD-L1 was shown to attenuate the induction of DNA double-strand breaks ( $\mathrm{pH} 2 \mathrm{AX}$ ) and caspase-3 cleavage by 5-fluorouracil (5-FU) and paclitaxel compared to parental CRC cells. Results were confirmed in PD-L1 knockout MC38 murine CRC cells where re-expression of wild-type PD-L1 promoted DNA damage and apoptosis. We also performed the clonogenic assay and flow cytometry to prove that loss of PD-L1 attenuated DNA damage and apoptosis induced by diverse anti-cancer drugs that could be reversed by restoration of wild-type PD-L1. Mechanistically, knockout of PD-L1 reduced chemosensitivity in association with reductions in p-AKT and in BH3-only proteins BIM and BIK, rather than STAT3 in CRC cells. However, STAT3 had a significant role in melanoma, which shows the heterogeneity of cancers. In summary, BRAFV600E can upregulate PD-L1 expression that was induced by c-jun and YAP to enhance chemotherapy-induced apoptosis. Together, we demonstrate a potential role for PD-L1 as a regulator of chemotherapy-induced apoptosis whose deletion or suppression confers chemoresistance. These findings expand the understanding of PD-L1 functions to include nonimmune mechanisms and suggest the potential use of PD-L1 as a biomarker of response to cytotoxic chemotherapy.
\end{abstract}

Key words: PD-L1, BRAF, apoptosis, therapeutic resistance, colorectal cancer

Colorectal cancers (CRC) with BRAFV600E are associated with poor prognosis and resistance to anti-cancer therapy. The BRAFV600E mutation substantially increases BRAF kinase activity, with subsequent phosphorylation and activation of MEK1 and MEK2. BRAFV600E mutations are shown to be significantly enriched in sporadic colon cancers with dMMR/MSI due to epigenetic inactivation of MLH1 $[1,2]$ that results in hypermutation, neoantigen production, and immune activation that creates an opportunity for immune checkpoint blockage. In this regard, anti-PD-1 monoclonal antibodies pembrolizumab and nivolumab are FDA approved for the treatment of advanced MSI-H CRC [3]. PD-1 is a cell surface receptor expressed by activated T-cells and macrophages, which can be bound by tumor cell PD-L1 that induces apoptosis of these immune cells
[4]. Tumor cells can evade immune surveillance via overexpression of the ligands of checkpoint receptors, e.g. PD-L1, on tumor cells or stromal cells, leading T cells to anergy or exhaustion [5]. Despite the overexpression of PD-L1, many cancers fail to respond to PD-1/PD-L1 inhibitors, suggesting that PD-L1 function in cancer is incompletely understood [6]. Recent evidence indicates that tumor cell PD-L1 may regulate intrinsic functions that include tumor cell apoptosis and autophagy $[7,8]$. PD-L1 may be a significant role for cell signaling effects that include regulation of tumor mammalian target of mTOR signaling [9], and alteration in mitogenactivated protein kinase (MAPK) [10].

The regulation of PD-L1 expression is likely complex with multiple signaling pathways involved. Factors that influence PD-L1 expression may also be dependent on cell types. For 
example, MEK/ERK (mitogen activated protein kinase/extracellular signal regulated kinase) signaling that is frequently activated in CRCs due to activating mutations in receptor tyrosine kinases such as the RAS GTPase or BRAF [11]. The BRAFV600E point mutation can be detected in $8 \%$ of human CRCs with activation of MEK1/2 by phosphorylation. That is attributed to resistance to anti-cancer therapy [12-15]. BRAFV600E is highly enriched in sporadic CRCs with microsatellite instability (MSI) [2], showing overexpression of $\mathrm{PD}-\mathrm{L} 1$, and frequent and durable response to anti-PD-1 antibodies [3]. BRAFV600E is a downstream effector of signal transduction mediated by the epidermal growth factor receptor (EGFR). Recent evidence suggests that EGFR activation can upregulate the expression of PD-L1, suggesting that BRAFV600E may regulate the expression of PD-L1 [16].

The development of resistance to chemotherapy and immunotherapy is a major obstacle in prolonging the survival of patients with cancer. Although several molecular mechanisms have been identified to contribute to chemoresistance $[17,18]$, the role of immune checkpoint molecules in the development of tumor chemoresistance has not been fully recognized. Traditionally, the emergence of chemoresistance and immunoresistance are considered as parallel and unrelated events. However, recent studies indicate that the overexpression of some immune checkpoint molecules (e.g. PD-L1) not only negatively influences antitumor immunity but also influence the resistance to chemotherapeutic agents [19-22]. Although the poor prognosis of PD-L1 positive tumors has been attributed to PD-L1's immune-suppressive function, the emerging role of PD-L1 in tumor chemoresistance may provide important clues in combating cancer.

In this report, we demonstrate that mutant BRAF can upregulate PD-L1 that is found to be expressed on CRC cells. The deletion of PD-L1 can reduce tumor cell growth shown by clonogenic assay. We examined PD-L1 expression in several human and mouse tumor cell lines in the context of their drug sensitivity in vitro and in vivo. We reported that overexpression of PD-L1 led to increased activation of ERK in tumor cells through an association with c-Jun and YAP, and PD-L1 deficiency renders tumor cells insusceptible to chemotherapy in a cell-context dependent manner. These findings expand the understanding of $\mathrm{PD}-\mathrm{L} 1$ functions to include nonimmune mechanisms and suggest the use of PD-L1 as a biomarker of response to cytotoxic chemotherapy such as fluoropyridine (5-FU).

\section{Materials and methods}

Gene expression analysis in TCGA RNA-Seq data. TCGA RNA-Seq of human colorectal cancers and associated somatic mutation data (in VCF format) together with the metadata for 478 samples and 41 normal colonic tissue samples were downloaded using the GDC data portal (https://gdc.cancer.gov/access-data/gdc-data-portal). The somatic mutation data were utilized to classify CRC cases into mutation for MSI-high, MSI-low, and MSS. The status of microsatellite instability (MSI) was previously described [23]. Log transformation of normalized gene expression in Fragments Per Kilobase of transcript per Million mapped reads (FPKM) was performed. The $\mathrm{R}$ ggplot2 package was utilized for data plotting.

Cell culture and reagents. BRAF mutant RKO and BRAF/ KRAS wild-type DiFi human colorectal cancer cell lines were obtained from the ATCC. VACO432 VT1 (BRAFWT/-) human CRC cell lines were obtained from Dr. B. Vogelstein [Genetic Resources Core Facility (GRCF), Johns Hopkins University, Baltimore, MD]. Parental and PD-L1 knockout B16F10 murine melanoma cells and MC38 murine CRC cells were obtained from Dong's lab [Immunology Department, Mayo Clinic, Rochester, MN]. All cell lines were tested and authenticated using short tandem repeat analysis. Cell lines were also routinely tested for Mycoplasma contamination every 3 months with a MycoAlert Mycoplasma detection set (Lonza). Cells were cultured as monolayers in RPMI medium (Invitrogen, catalog no. 11875) with supplementation of $10 \%$ FBS and 1\% antibiotic-antimycotic (Invitrogen, catalog no. 15240). Lentivirus producer cells, HEK293T, were grown in high-glucose DMEM (Sigma, catalog no. D5796) that was supplemented as mentioned above.

Cells were treated with cobimetinib (GDC-0973/XL-518; Active Biochem, catalog no. A-1180), dinaciclib (Sellekchem, catalog no. S2768), CPT-11 (Sigma), oxaliplatin (Sigma), or fluoropyridine (Sigma) at indicated doses and times. Paclitaxel was obtained from Dong's lab [Immunology Department, Mayo Clinic, Rochester, MN]. Anti-mouse PD-L1 (10F.9G2, Bioxcell, West Lebanon, NH) antibody treatment was performed in the Opti-Mem medium. Drugs were dissolved in DMSO, prepared as stock solutions, aliquoted, and then stored at $-20^{\circ} \mathrm{C}$. Drugs were diluted in growth medium at the time of treatment. For immunoblotting, all primary antibodies were purchased from Cell Signaling Technology.

Lentiviral CRISPR knockout, mutagenesis, and ectopic gene expression. Human PD-L1 guide RNA (target sequence TACCGCTGCATGATCAGCTA) cloned in lentiviral vector pLentiCRISPRv2 was purchased from Genscript. Human PD-L1 cDNA template was obtained from Origene (catalog no. sc115168), which was subcloned into vector pCDH1puro-2HA. Generation of PD-L1 deletion mutants of intracellular or extracellular domains was performed using specific PCR primers. Production and transduction of lentivirus into target cells and the elimination of non-transduced target cells were performed using our standard procedure [24].

siRNA transfection. YAP and c-JUN siRNA were purchased from Santa Cruz Biotechnology (catalog no. sc-38637) and Cell Signaling Technology (catalog no. \#6204); AllStars Negative Control siRNA was obtained from Qiagen (catalog no. SI03650318). YAP and c-JUN siRNA at 100 $\mathrm{nmol} / \mathrm{l}$, alone or in combination, were transfected into RKO cells, as previously described [25]. Briefly, Lipofectamine RNAi Max (Invitrogen, catalog no. 13778150) and siRNA 
were each diluted in Opti-MEM medium (Invitrogen), which were then combined to form siRNA-lipid complex and added to target cells that were grown in. After treatment, floating cells in growth medium were combined with adherent cells that were detached using TrypLE ${ }^{\mathrm{mi}}$ Express Enzyme (ThermoFisher Scientific, catalog no. 12604013). Cells were then washed 3 times in cold PBS, resuspended in $1 \times$ Annexin $\mathrm{V}$ binding solution (BD Biosciences, catalog no. 556454), and stained with Annexin V conjugated with FITC (BD Biosciences, catalog no. 556419). Apoptotic cells were quantified by flow cytometry. Results were imported into Matlab (Mathworks) and processed antibiotic-free medium at $30-50 \%$ confluence at the time of transfection. Knockdown efficiency was verified 48 hours post-transfection.

Apoptosis assay. After treatment, floating cells in the growth medium were combined with adherent cells that were detached using TrypLE ${ }^{\mathrm{su}}$ Express Enzyme (Thermo Fisher Scientific, catalog no. 12604013). Cells were then washed 3 times in cold PBS, resuspended in $1 \times$ Annexin V binding solution (BD Biosciences, catalog no. 556454), and stained with Annexin V conjugated with FITC (BD Biosciences, catalog no. 556419). Apoptotic cells were quantified by flow cytometry. Results were imported into Matlab (Mathworks) and processed.

Immunoblotting. Protein lysates were prepared in a lysis buffer [ $5 \mathrm{mmol} / \mathrm{l} \mathrm{MgCl}, 137 \mathrm{mmol} / \mathrm{l} \mathrm{KCl}, 1 \mathrm{mmol} / \mathrm{l} \mathrm{EDTA}$, $1 \mathrm{mmol} / \mathrm{l}$ EGTA, 1\% CHAPS, $10 \mathrm{mmol} / \mathrm{l} \mathrm{HEPES} \mathrm{(pH} \mathrm{7.5)]}$ with supplementation of a protease inhibitor cocktail and a phosphatase inhibitor cocktail 2 (both from Sigma), which were then normalized using NanoDrop measurement (NanoDrop Technologies) or Bio-Rad protein assay (catalog no. 500-0006). After being denatured in LDS sample buffer (Invitrogen) supplemented with 2-mercaptoethanol (Bio-Rad), protein samples were loaded onto $10 \%$ or $14 \%$ SDS-PAGE gels which were then transferred electrophoretically onto a polyvinylidene difluoride membrane (Bio-Rad). The membrane was blocked with $0.2 \%$ I-Block (Applied Biosystems) in PBS-T (PBS containing 0.1\% Tween 20) and incubated with the primary antibodies in PBS-T containing $0.2 \%$ I-Block overnight at $4{ }^{\circ} \mathrm{C}$ or at room temperature for 3 hours. The membranes were then washed and incubated with a secondary antibody in PBS-T containing $0.2 \%$ I-Block conjugated to alkaline phosphatase, followed by development with CDP-Star substrate (Applied Biosystems).

Clonogenic assay. Cells were inoculated at a density of 200 cells/well in 6-well plates. After attachment, a fresh growth medium was added and cells were allowed to grow for 8-10 days. Cells colonies were visualized by fixation in $10 \%$ methanol $/ 10 \%$ acetic acid and stained with $0.5 \%$ crystal violet in $10 \%$ methanol. Each condition was performed in triplicate. The colony area was estimated using the ImageJ plugin ColonyArea [26].

Competitive RT-PCR. Total RNA was extracted from parental or PD-L1 knockout MC38 cells with or without re-expression of human wild-type PD-L1 or its deletion mutants of intracellular of the extracellular domain. Competitive RT-PCR was performed with a one-step RT-PCR kit (Qiagen) using the following primer sets containing a 4:1 molar ratio of PD-L1 (forward: TGCTGCATAATCAGCTACGG; reverse: TCCACGGAAATTCTCTGGTT) against $\beta$-actin (forward, 5'-GTGACGTTGACATCCGTAAAGA-3'; reverse, 5'-GCCGGACTCATCGTACTCC-3'). Reverse transcription was coupled with PCR (25 cycles) on a thermocycler (Applied Biosystems, Grand Island, NY). PCR products were quantified on the Agilent Bioanalyzer 2000 using the DNA 1,000 kit. In brief, samples were loaded onto DNA microchips, and the DNA fragments were then separated by capillary electrophoresis. The target DNA sizes and relative quantities were calculated on the basis of DNA ladders and an internal marker, respectively. The associated software then generates agarose gel-like images.

Statistical analysis. Annexin V data in cell culture experiments and clonogenic survival assays were expressed as mean \pm SD. All cell culture experiments were performed in triplicate. Student t-test (two-tailed) was performed for statistically significant with $\alpha<0.05$ as the cut-off.

\section{Results}

BRAFV600E upregulates PD-L1 expression in colorectal cancer cells. We determined the potential association of BRAFV600E with PD-L1 expression utilizing TCGA RNA-Seq and mutational data from CRCs. In the previous study, we found that BRAFV600E vs. non- BRAFV600E (including normal colonic, KRAS mutant, or wild-type for both BRAF and KRAS) cases showed upregulation of PD-L1 mRNA expression. BRAFV600E was shown to be enriched in MSI-H vs. MSI-L or MSS CRCs as is well described (Figure 1A). The upregulation of PD-L1 was associated with an increase in the transcription factor YAP, a Hippo effector, which are downstream effectors of MAPK signaling. We found that YAP expression is associated with PD-L1 expression (Figure 1B). Furthermore, c-JUN and YAP were found to cooperatively regulate $\mathrm{PD}-\mathrm{L} 1$ expression that is consistent with the reported ability of YAP to mediate PD-L1 expression in BRAF inhibitor-resistant human melanoma cell lines [27].

CRC cell lines with BRAF or KRAS mutations showed variable PD-L1 protein expression (Figure 1C). Expression of pERK, a known downstream phosphorylation event of BRAFV600E, also correlated with BRAFV600E allele copy number [22]. Parental RKO cells contain two copies of BRAFV600E and had the most abundant PD-L1 expression. As for MSS (DiFi) or MSI (Vaco432 VT1) cell line, ectopic BRAFV600E was also shown to induce the expression of PD-L1 and the c-JUN transcription factor that is a downstream target of MEK/ERK signaling (Figure 1D). KRAS mutation can upregulate PD-L1 expression in HCT116 cells as well. The upregulation of PD-L1 by BRAFV600E was due to the increased gene transcription as shown by a competitive reverse transcriptase-PCR (RT-PCR) assay (Figure 1E). 
A

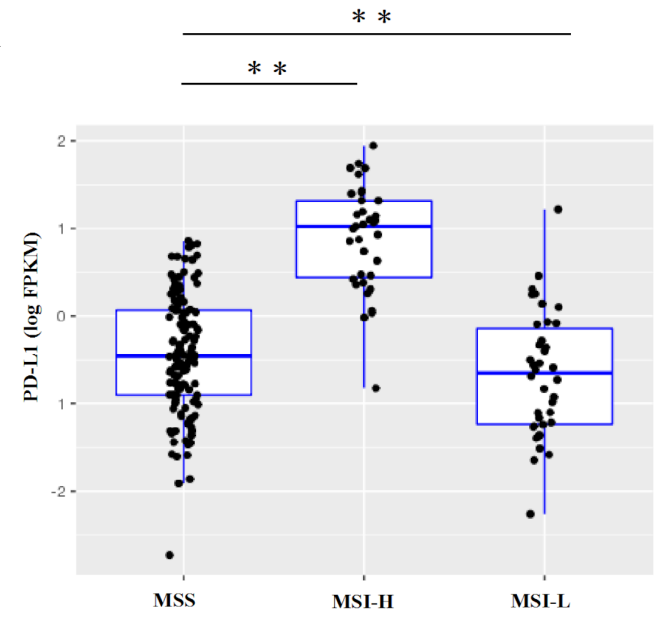

C

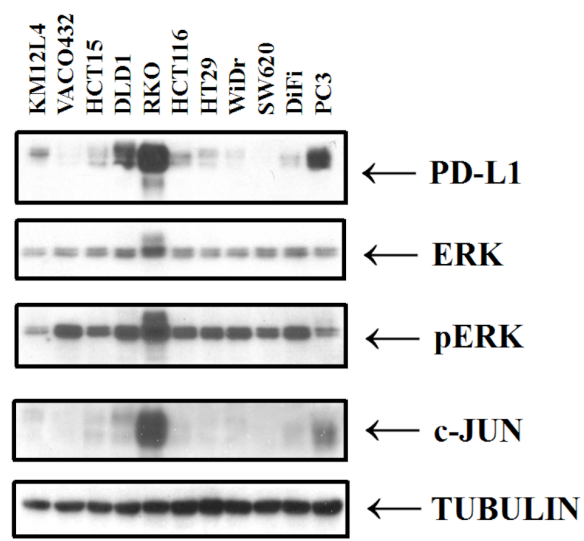

$\mathbf{E}$
B

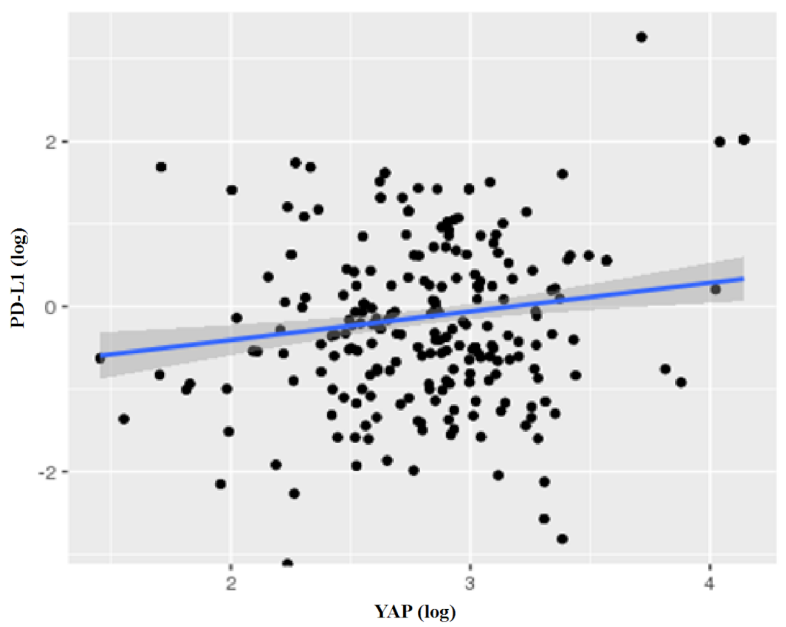

D

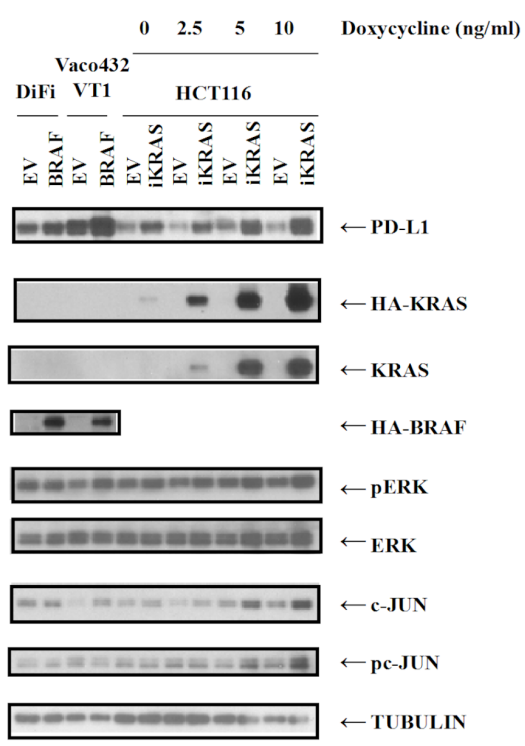

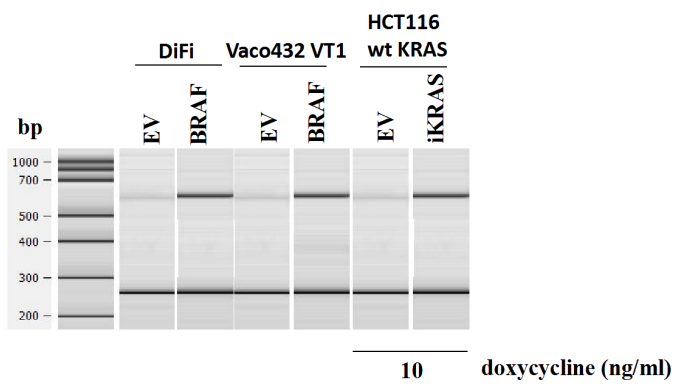

Figure 1. The potential association of BRAFV600E with programmed death ligand 1 (PD-L1) expression utilizes TCGA RNA-Seq and mutational data from colorectal cancer (CRC) cells. A) PD-L1 (CD274) gene expression data were extracted from TCGA RNA-seq datasets for colon cancers that were categorized based on MSI-high expression ( $n=31)$, MSI-low expression $(n=35)$, or MSS expression $(n=385)$ using associated metadata; mRNA expression was compared among these colon cancer subtypes. Statistical significance was calculated using a two-way analysis of variance (ANOVA). ${ }^{* *} \mathbf{p}<0.01$ B) PD-L1 (CD274) gene expression and YAP expression were extracted from TCGA RNA-seq datasets for colon cancers. Statistical significance was calculated using a two-way analysis of variance (ANOVA). The Pearson's product-moment correlation is 0.1648838 . ${ }^{* *}<0.01$. C) $\mathrm{PD}-\mathrm{L} 1$ protein expressions were examined in multiple human CRC cell lines by immunoblotting. PC3 is a prostate cancer cell line as a positive control. The expression of $\beta$-tubulin served as a loading control. D) DiFi and Vaco432 VT1 cell lines containing ectopic BRAFV600E or empty vector (EV) were compared for the expression of PD-L1, p-ERK, ERK, pc-JUN, and c-JUN. PD-L1 protein expression was examined in HCT116 cells with doxycycline-inducible mutant KRAS (iKRAS). E) Competitive reverse transcriptase-PCR (RT-PCR) was performed to compare PD-L1 messenger RNA (mRNA) among isogenic cells or those with ectopic BRAFV600E/-/- or inducible KRAS system vs. empty vector. 
MEK/ERK inhibitor attenuates PD-L1 expression. Given that both mutations in BRAF and KRAS can upregulate PD-L1 expression, we tested the effects of MEK/ERK inhibition that is downstream of the RAF/RAF cascade. Using the MEK/ERK inhibitor cobimetinib that blocked pERK expression, we observed a dose-dependent reduction in PD-L1 expression in RKO cells (Figure 2A) or in Vaco432 VT1 cells with ectopic BRAFV600E vs. empty vector (Figure 2B). Cobimetinib was also shown to downregulate PD-L1 expression in a dose-dependent manner in DiFi cells with ectopic BRAFV600E (Figure 2A). In contrast to that, CDK inhibitor dinaciclib failed to do so (Figure 2B). Co-regulation of PD-L1 by both c-JUN and YAP was confirmed by the ability of combined suppression of these two transcription factors to reduce PD-L1 expression to a greater extent than their individual knockdown by siRNA or shRNA (Figure 2C). We also found that STAT3 knockdown cannot downregulate PD-L1 expression in CRC cells (Figure 2D). ERK or c-JUN siRNA was shown to reduce PD-L1 expression (Figure 2E).
Knockout of the PD-L1 gene displays resistance to chemotherapy-mediated apoptosis. While tumor cells can evade immune surveillance due to overexpression of checkpoint proteins, e.g. PD-L1, on tumor cells or adjacent cells leading $\mathrm{T}$ cells to anergy or exhaustion [5], the function of tumor cell-intrinsic PD-L1 expression on tumor cells is poorly understood. Given that tumor cell escape from destruction by apoptosis is one of the hallmarks of malignancy, we investigated the effect of PD-L1 on anti-cancer drug-induced tumor cell apoptosis by generating PD-L1 knockout cells. Given that tumor cell resistance to apoptosis is one of the hallmarks of malignancy [28], we determined the effect of tumor cell PD-L1 on apoptosis induced by diverse chemotherapy in human CRC cells with the knockout of PD-L1 by CRISPRCas9 genome editing. In the previous study, the knockout of PD-L1 in RKO cells was shown to markedly reduce the induction of apoptosis (cleaved caspase-3) and DNA doublestrand breaks $(\mathrm{pH} 2 \mathrm{Ax})$ by treatment with irinotecan or oxaliplatin [22]. This finding was not limited to two drugs
A

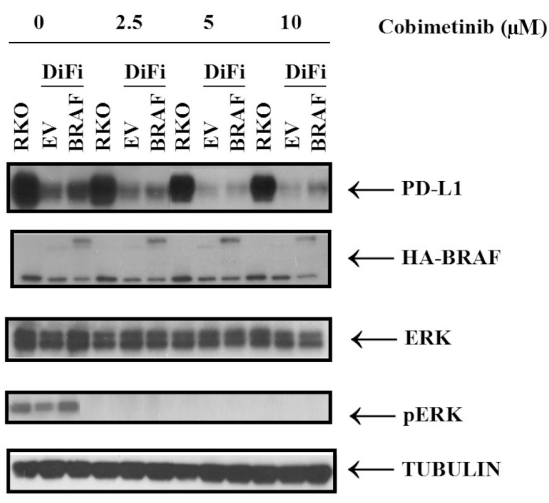

B

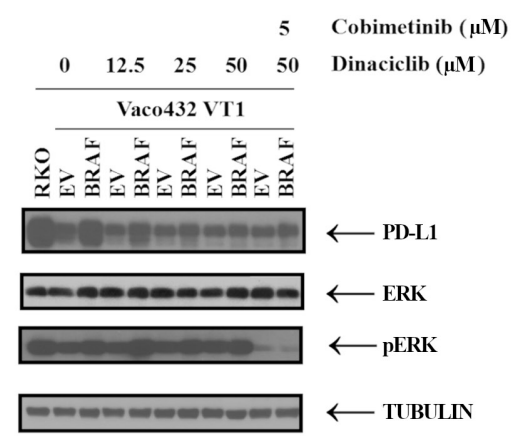

E

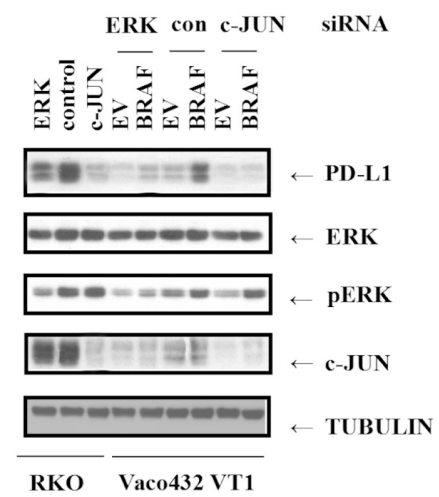

Figure 2. Inhibition of MEK/ERK signaling downregulates BRAFV600E-induced programmed death ligand 1 (PD-L1) expression. A) RKO cell lines or DiFi cells with ectopic BRAFV600E or empty vector (EV) were treated with cobimetinib and compared for protein expression of PD-L1 by immunoblotting. B) Expression of designated proteins was examined in Vaco432 VT1 cells with ectopic BRAFV600E or with EV that were treated with dinaciclib and cobimetinib. C) RKO cells with YAP shRNA were transfected with c-JUN siRNA, and the expression of indicated proteins was determined by immunoblotting. D) Knockdown of STAT3 cannot attenuate PD-L1 expression in RKO cells. E) RKO and Vaco432 VT1 with ectopic BRAF cells were transfected with ERK or c-JUN small interfering RNA (siRNA), and cell lysates were immunoblotted with the indicated antibodies. 
A

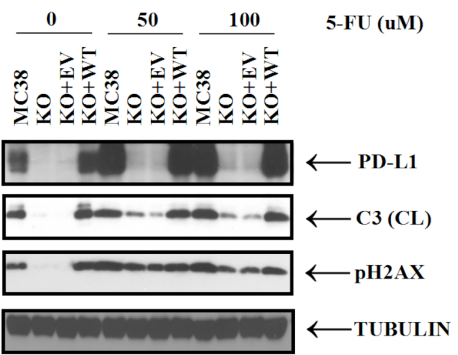

B

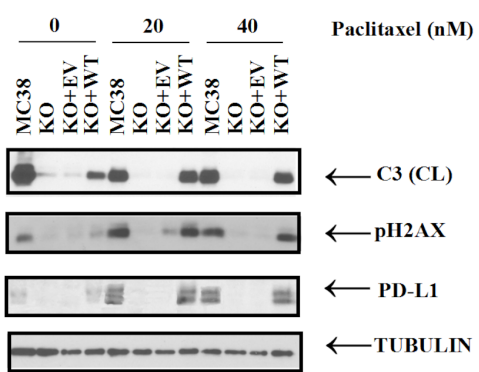

C
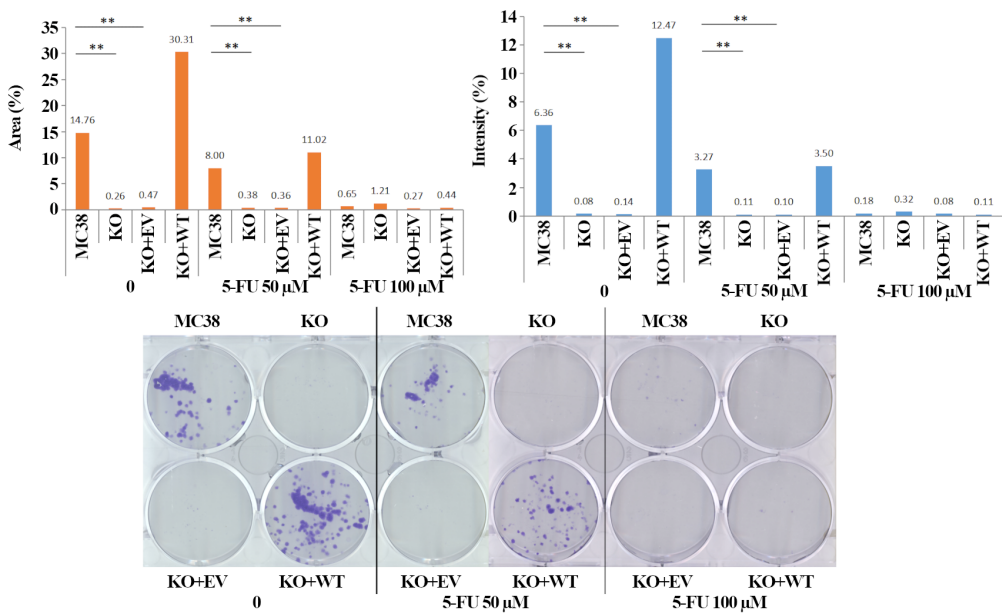

D
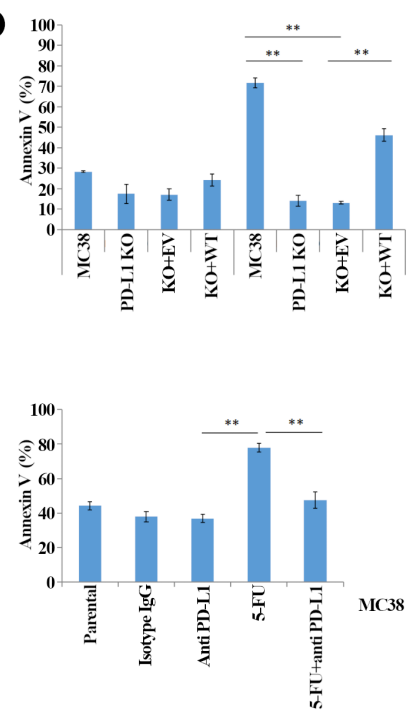
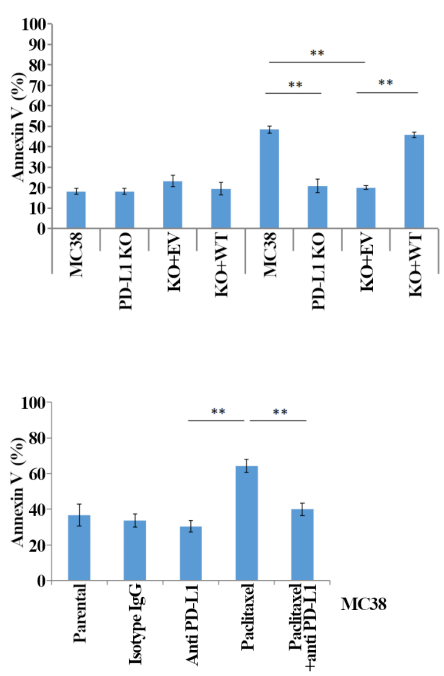

E

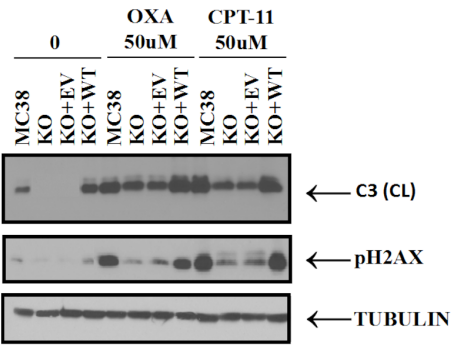

Figure 3. Programmed death ligand 1 (PD-L1) knockout (KO) in CRCs confers resistance to chemotherapy-induced apoptosis and DNA double-strand breaks (DSBs) that can be reversed by re-expression of PD-L1, but not its deletion mutants. A) PD-L1-KO MC38 cells with stable ectopic expression of human PD-L1 were generated. The MC38 cell line and its derivatives were treated with 5-FU for $48 \mathrm{~h}$, and the expression of pH2AX and cleaved (CL) caspase-3 (C3) was determined. The data are presented as mean \pm S.E.M. of $\mathbf{n}=3$ independent experiments. B) The MC 38 cell line and its derivatives were treated with paclitaxel for $48 \mathrm{~h}$, and the expression of $\mathrm{pH} 2 \mathrm{AX}$ and cleaved (CL) caspase-3 (C3) was determined. The data are presented as mean \pm S.E.M. of $n=3$ independent experiments. C) The ability of the MC38, PD-L1 KO, or PD-L1 KO cells with re-expression of wild-type (WT) PD-L1 or empty vector (EV) to form colonies was determined using a clonogenic assay. The MC38 cell line and its derivatives were also treated with CPT-11 or oxaliplatin for $16 \mathrm{~h}$ using the clonogenic assay. The data are presented as mean \pm S.E.M. of $n=3$ independent experiments. Statistical significance was calculated using two-way ANOVA, ${ }^{* *} \mathrm{p}<0.01$. D) The MC38 cell line and its derivatives were treated with 5 -FU or paclitaxel for $48 \mathrm{~h}$, and the expression of $\mathrm{pH} 2 \mathrm{AX}$ and cleaved (CL) caspase-3 (C3) was determined. MC38 cells were treated with 5-FU (100 $\mu \mathrm{M})$ or paclitaxel $(4 \mu \mathrm{g} / \mathrm{ml})$ in the presence or absence of anti-PD-L1 antibody $(40 \mu \mathrm{g} / \mathrm{ml})$. Annexin $\mathrm{V}+$ apoptotic cells were quantified. The data are presented as mean $\pm S$.E.M. of $n=3$ independent experiments. Statistical significance was calculated using two-way ANOVA. ${ }^{*} \mathrm{p}<0.01$. E) The MC38 cell line and its derivatives were treated with CPT11 or oxaliplatin for $24 \mathrm{~h}$, and the expression of $\mathrm{pH} 2 \mathrm{AX}$ and cleaved (CL) caspase-3 (C3) was determined. The data are presented as mean \pm S.E.M. of $\mathrm{n}=3$ independent experiments. 
since PD-L1 knockout in murine MC38 colon cancer cells also reduced induction of apoptosis and DNA damage by 5-FU or paclitaxel (Figures 3A, 3B). 5-FU can induce PD-L1 expression. We also found that PD-L1 knockout MC38 cells displayed a slower growth rate, as shown by reduced colony size in clonogenic assay, which could be restored by re-expression of a human wild-type PD-L1 (Figure 3C). The results were consistent with reduced caspase- 3 cleavage and Annexin V-labeling (Figure 3D). Furthermore, treatment with the anti-PD-L1 antibody for MC38 cells attenuated 5 -FU or paclitaxel-induced apoptosis, which was demonstrated by Annexin V labeling (Figure 3D).

To confirm this finding, we utilized parental and PD-L1 knockout in murine B16F10 melanoma cells. We then compared chemotherapy-induced apoptosis among B16F10 and its derivative cells. Similar to our observations in MC38 cells (Figure 3E), knockout of PD-L1 conferred resistance to irinotecan, oxaliplatin, or cobimetinib-induced DNA double-strand breaks and apoptosis (Figures 4A, 4B).

\section{Discussion}

In this report, we studied whether PD-L1 can be regulated by $\mathrm{V} 600 \mathrm{E}$ oncogenic driver mutation in BRAF. Using TCGA data, we previously demonstrated an increase in PD-L1 mRNA expression in human colon cancer patients with BRAFV600E compared to non-mutated BRAF [22]. However, BRAFV600E is enriched in CRCs with MSI-high $[1,2]$. Thus, we confirmed MSI-high CRCs show increased PD-L1 mRNA expression. We observed that BRAFV600E could increase the expression of PD-L1 in MSS DiFi cell lines, indicating that PD-L1 expression was not limited to MSI CRC cells. We found that overexpression of PD-L1 resulted in increased activation of the ERK pathway in CRC cells. The induction of PD-L1 protein expression could be suppressed by a MEK inhibitor.

Upregulation of PD-L1 was associated with an increase in the transcription factors C-JUN and YAP (a Hippo effector), which are inducible transcription factors that direct changes of gene expression such as PD-L1 [29], suggesting that these 2 kinases may be involved in the modulation of PD-L1 in response to multiple extracellular stimuli. Either the inhibition of the activity of its upstream kinase MEK with MEKi (U0126) or the physical knockdown of ERK1/2 or JNK are able to reduce PD-L1 expression in melanoma cells [30-33]. In addition, we found that c-JUN and YAP synergistically regulated the expression of $\mathrm{PD}-\mathrm{L} 1$, which was consistent with the reported ability of YAP-mediated expression of PD-L1 in BRAF-resistant human melanoma cell lines [27]. However, we further observed that a knockdown of STAT3 did not inhibit the expression of PD-L1. The results indicated that cooperation of c-JUN and YAP factors in the modulation of PD-L1 expression in the CRC cells. Mechanistically, YAP forms a complex with TEAD and binds to the promoter of PD-L1 through the DNA binding domain [34]. ERK activation has been shown to increase the expression and activity of c-JUN [35], and MEK inhibition inhibits PD-L1 transcription in multiple myeloma cells $[30,36]$. The upregulation of PD-L1 in melanoma cells resistant to BRAF or MEK inhibitors is partly due to post-transcriptional mechanisms [37].

In addition, the mutations of BRAF, NRAS, PTEN, or AKT had no correlation with the expression level of PD-L1
A
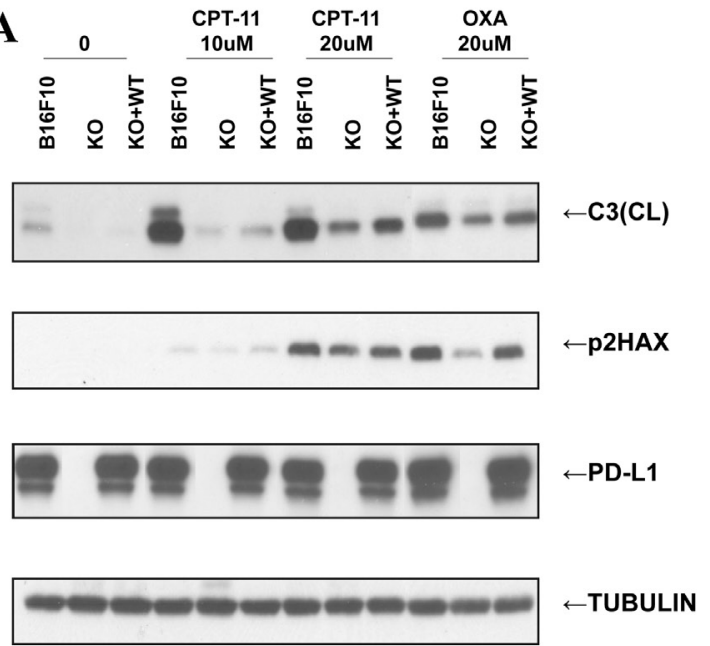

B
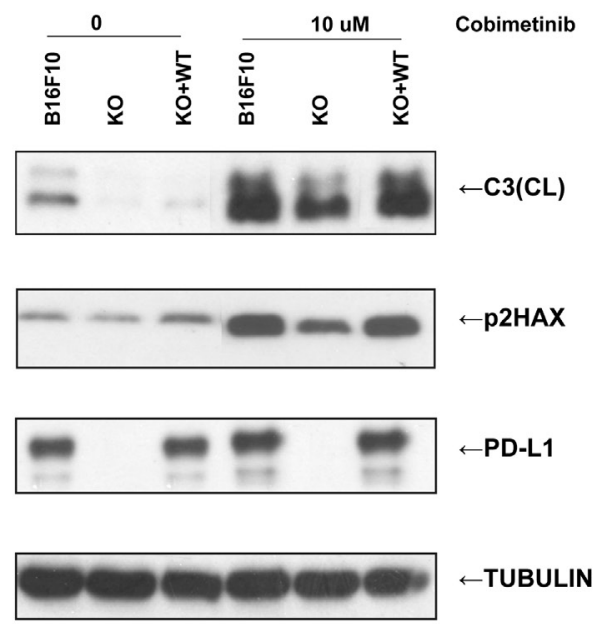

Figure 4. Programmed death ligand 1 (PD-L1) knockout (KO) in melanoma cells confers resistance to chemotherapy-induced apoptosis and DNA double-strand breaks (DSBs) that can be reversed by re-expression of PD-L1, but not its deletion mutants. A) PD-L1-KO B16F10 cells with stable ectopic expression of human PD-L1 were generated. The B16F10 cell line and its derivatives were treated with CPT-11 or oxaliplatin for 24 h, and the expression of $\mathrm{pH} 2 \mathrm{AX}$ and cleaved $(\mathrm{CL})$ caspase-3 $(\mathrm{C} 3)$ was determined. The data are presented as mean $\pm \mathrm{S}$.E.M. of $\mathrm{n}=3$ independent experiments. B) The B16F10 cell line and its derivatives were treated with cobimetinib for $24 \mathrm{~h}$, and the expression of pH2AX and cleaved (CL) caspase-3 (C3) was determined. The data are presented as mean \pm S.E.M. of $n=3$ independent experiments. 
in melanoma cell lines, suggesting that there were differences in tumor cell types [38]. Preclinical studies have shown that BRAF inhibition leads to rapid feedback activation of EGFR, which supports the continued proliferation of EGFR in the presence of BRAF inhibition [39]. In a clinical trial, after BRAFV600E CRCs patients were treated with BRAF, EGFR, and MEK inhibitors, MAPK was more strongly inhibited for improving the efficacy [40]. Our data suggest that the expression of PD-L1 in tumor cells can be upregulated by BRAF activation to enhance chemotherapy-induced apoptosis. To establish a link between EGFR and PD-L1 in CRC cells, it is meaningful to study the effect of EGFR antagonism on PD-L1 levels.

To date, there are few studies on the intrinsic PD-L1 or PD-1 of tumor cells and its non-immune function in human cancer cells. We found that the growth rate of CRC cells with PD-L1 knockout was slower than that of parental cells including after implantation in nude mice, and ectopic PD-L1 expression could enhance this growth rate. These data suggest that this effect occurs in the absence of a functional adaptive immune system. Consistent with our finding, inhibition of PD-L1 defects in ovarian and melanoma cancer cell lines reduced their proliferation and tumor formation delays compared with normal and immunodeficient NSG mice $[8,9]$.

Data for the regulation of apoptotic susceptibility by PD-L1 are derived from a limited number of studies in solid tumor cell lines [20, 41, 42]. We found that the intrinsic PD-L1 of tumor cells can regulate chemosensitivity, and knockout of PD-L1 can make human and mouse CRC cell lines resist the apoptosis induced by various cytotoxic drugs. Furthermore, anti-PD-L1 antibodies can attenuate the chemotherapyinduced apoptosis, which was consistent with the effects of PD-L1 knockout. Since genome editing by CRISPR/Cas9 may produce off-target effects [43], we demonstrated that ectopic wild type PD-L1 can restore drug-induced apoptotic sensitivity. As ERK activation plays key roles in cell survival and drug resistance [44, 45], the increased ERK activation in PD-L1 overexpressing tumor cells suggests that PD-L1 may promote the ERK pathway in tumor cells to survive upon cytotoxic chemotherapy. We confirmed our data in a xenograft model of immunodeficient mice in which tumors produced by PD-L1 knockout cells showed resistance to 5-FU-induced tumor regression compared with tumors treated with vehicle drugs. It has been reported that PD-L1 has a function beyond the negative regulation of $\mathrm{T}$ cell responses through its receptor PD-1 [46]. Ligation of PD-L1 by antibody causes PD-L1 internalization and disrupts the role of PD-L1 in the maintenance of Akt/mTOR signaling [46]. To that end, Kleffel et al. identified that expression of PD-1 in melanoma cells modulates downstream mTOR signaling [9]. In contrast to our previous studies, human RKO and murine MC38 colon cancer cells lack endogenous PD-1 expression. Therefore, our data suggest that PD-L1 regulates chemotherapy-induced apoptosis. In contrast to our data, downregulation of PD-L1 has been shown to make breast cancer, small cell lung cancer, and lymphoma cells sensitive to chemotherapy-induced apoptosis. The reported differences suggest that these findings are tumor cell type-specific.

Of note, PD-L1-mediated chemoresistance is cellcontext dependent based on the drug selection. We found that knocking out PD-L1 sensitizes tumor cells (RKO and MC38) with high steady state expression of PD-L1 protein became less susceptible to 5 -FU more so than parental cells. These results suggest that high expression of PD-L1 may build a scaffold to maintain high activation of the MAPK pathway that contributes to retaining a high level of Bcl-2 [47], an anti-apoptosis molecule. By analyzing the regulation of PD-L1 on Bcl-2 family proteins, the mechanism of PD-L1 regulating cell apoptosis was further understood. In our previous study, we observed that in PD-L1 knockout RKO and MC38 cells, only the pro-apoptotic proteins BIM and $\mathrm{BIK}$ of $\mathrm{BH} 3$ protein were reduced, while the reexpression of wild type PD-L1 reversed this change. BIM has been shown to directly activate BAX and BAK, which can result in their homo-oligomerization, thus promoting mitochondrial membrane permeabilization and apoptosis [48]. We also found that knockout of PD-L1 can downregulate p-AKT, and it has been reported that inhibition of AKT can activate FoxO transcription factors and enhance the expression of target genes BIM. However, AKT inhibition did not significantly alter the expression of BIM or BIK in CRC cells.

In summary, we found that BRAFV600E could upregulate the expression of PD-L1 by MAPK signaling pathway via enhancing the activity of c-JUN and YAP, thus inducing BIM and BIK proteins to enhance chemotherapy-induced apoptosis. The clinical implication of our study is that the status of PD-L1 expression by cancer cells should be considered in optimizing chemotherapy in order to overcome PD-L1 mediated chemoresistance. These data suggest that the intrinsic PD-L1 effect in CRC cells is non-immune regulation, suggesting that PD-L1 has a broader role as a potential biomarker for predicting cancer response to treatment.

Acknowledgments: We express our gratitude to Mr. Matthew A. Bockol for downloading the TCGA data.

\section{References}

[1] Cancer Genome Atlas Network. Comprehensive molecular characterization of human colon and rectal cancer. Nature 2012; 487: 330-337. https://doi.org/10.1038/nature11252

[2] Weisenberger DJ, Siegmund KD, Campan M, Young J, Long TI et al. CpG island methylator phenotype underlies sporadic microsatellite instability and is tightly associated with BRAF mutation in colorectal cancer. Nat Genet 2006; 38: 787-793. https://doi.org/10.1038/ng1834

[3] Le DT, Hubbard-Lucey VM, Morse MA, Heery CH, Dwyer A et al. A Blueprint to Advance Colorectal Cancer Immunotherapies. Cancer Immunol Res 2017; 5: 942-949. https:// doi.org/10.1158/2326-6066.CIR-17-0375 
[4] Zou W, Chen L. Inhibitory B7-family molecules in the tumour microenvironment. Nat Rev Immunol 2008; 8: 467477. https://doi.org/10.1038/nri2326

[5] Wang Y, Wu L, Tian C, Zhang Y. PD-1-PD-L1 immunecheckpoint blockade in malignant lymphomas. Ann Hematol 2018; 97: 229-237. https://doi.org/10.1007/s00277-0173176-6

[6] Hamanishi J, Mandai M, Matsumura N, Abiko K, Baba T et al. PD-1/PD-L1 blockade in cancer treatment: perspectives and issues. Int J Clin Oncol 2016; 21: 462-473. https://doi. org/10.1007/s10147-016-0959-Z

[7] Azuma T, Yao S, Zhu G, Flies AS, Flies SJ et al. PD-L1 is a ubiquitous antiapoptotic receptor on cancer cells. Blood 2008; 111: 3635-3643. https://doi.org/10.1182/ blood-2007-11-123141

[8] Clark CA, Gupta HB, Sareddy G, Pandeswara S, Lao S et al. Tumor-intrinsic PD-L1 signals regulate cell growth, pathogenesis, and autophagy in ovarian cancer and melanoma. Cancer Res 2016; 76: 6964-6974. https://doi. org/10.1158/0008-5472.CAN-16-0258

[9] Kleffel S, Posch C, Barthel SR, Mueller H, Schlapbach C et al. Melanoma cell-intrinsic PD-1 receptor functions promote tumor growth. Cell 2015; 162: 1242-1256. https://doi. org/10.1016/j.cell.2015.08.052

[10] Orzechowski A, Dong H. PD-L1 confers tumor chemoresistance by regulating MAPK/ERK activation. Cancer Res. 2014; 74: abstract 5026. https://doi.org/10.1158/1538-7445. AM2014-5026

[11] Roberts PJ, Der CJ. Targeting the Raf-MEK-ERK mitogen activated protein kinase cascade for the treatment of cancer. Oncogene 2007; 26: 3291-3310. https://doi.org/10.1038/ sj.onc. 1210422

[12] Phipps AI, Ahnen DJ, Cheng I, Newcomb PA, Win AK et al. PIK3CA somatic mutation status in relation to patient and tumor factors in racial/ethnic minorities with colorectal cancer. Cancer Epidemiol Biomarkers Prev 2015; 24: 1046-1051. https://doi.org/10.1158/1055-9965.EPI-15-0204

[13] Zaanan A, Okamoto K, Kawakami H, Khazaie K, Huang S et al. The mutant KRAS gene up-regulates BCL-XL protein via STAT3 to confer apoptosis resistance that is reversed by BIM protein induction and BCL-XL antagonism. J Biol Chem 2015; 290: 23838-23849. https://doi.org/10.1074/jbc. M115.657833

[14] Kopetz S, Desai J, Chan E, Hecht JR, O’Dwyer PJ et al. Phase II pilot study of vemurafenib in patients with metastatic BRAF-mutated colorectal cancer. J Clin Oncol 2015; 33: 4032-4038. https://doi.org/10.1200/JCO.2015.63.2497

[15] Missiaglia E, Jacobs B, D’Ario G, Di Narzo AF, Soneson C et al. Distal and proximal colon cancers differ in terms of molecular, pathological, and clinical features. Ann Oncol 2014; 25: 1995-2001. https://doi.org/10.1093/annonc/mdu275

[16] Chen N, Fang W, Zhan J, Hong S, Tang Y et al. Upregulation of PD-L1 by EGFR activation mediates the immune escape in EGFR-driven NSCLC: implication for optional immune targeted therapy for NSCLC patients with EGFR mutation. J Thorac Oncol 2015; 10: 910-923. https://doi.org/10.1097/ JTO.0000000000000500
[17] Brozovic A, Osmak M. Activation of mitogen-activated protein kinases by cisplatin and their role in cisplatin-resistance. Cancer Lett 2007; 251: 1-16. https://doi.org/10.1016/j.canlet.2006.10.007

[18] McCubrey JA, Steelman LS, Chappell WH, Abrams SL, Wong EW et al. Roles of the Raf/MEK/ERK pathway in cell growth, malignant transformation and drug resistance. Biochim Biophys Acta 2007; 1773: 1263-1284. https://doi.org/10.1016/j. bbamcr.2006.10.001

[19] Tamura H, Ishibashi M, Yamashita T, Tanosaki S, Okuyama $\mathrm{N}$ et al. Marrow stromal cells induce PD-L1 expression on myeloma cells, generating aggressive characteristics in multiple myeloma. Leukemia 2013; 27: 464-472. https://doi. org/10.1038/leu.2012.213

[20] Ghebeh H, Lehe C, Barhoush E, Al-Romaih K, Tulbah A et al. Doxorubicin downregulates cell surface PD-L1 expression and upregulates its nuclear expression in breast cancer cells: role of PD-L1 as an anti-apoptotic molecule. Breast Cancer Res 2010; 12: R48. https://doi.org/10.1186/bcr2605

[21] Liu H, Tekle C, Chen YW, Kristian A, Zhao Y et al. B7-H3 silencing increases paclitaxel sensitivity by abrogating Jak2/ Stat3 phosphorylation. Mol Cancer Ther 2011; 10: 960-971. https://doi.org/10.1158/1535-7163.MCT-11-0072

[22] Feng D, Qin B, Pal K, Sun L, Dutta S et al. BRAFV600E-induced, tumor intrinsic PD-L1 can regulate chemotherapyinduced apoptosis in human colon cancer cells and in tumor xenografts. Oncogene 2019; 38: 6752-6766. https://doi. org/10.1038/s41388-019-0919-y

[23] Kim TM, Laird PW, Park PJ. The landscape of microsatellite instability in colorectal and endometrial cancer genomes. Cell 2013; 155: 858-868. https://doi.org/10.1016/j. cell.2013.10.015

[24] Kawakami H, Huang S, Pal K, Dutta SK, Mukhopadhyay D et al. Mutant BRAF upregulates MCL-1 to confer apoptosis resistance that is reversed by MCL- 1 antagonism and cobimetinib in colorectal cancer. Mol Cancer Ther 2016; 15: 30153027. https://doi.org/10.1158/1535-7163.MCT-16-0017

[25] Zhang P, Kawakami H, Liu W, Zeng X, Strebhardt K et al. Targeting CDK1 and MEK/ERK Overcomes Apoptotic Resistance in BRAF-Mutant Human Colorectal Cancer. Mol Cancer Res 2018; 16: 378-389. https://doi.org/10.1158/15417786.MCR-17-0404

[26] Guzman C, Bagga M, Kaur A, Westermarck J, Abankwa D. Colony area: an ImageJ plugin to automatically quantify colony formation in clonogenic assays. PLoS One 2014; 9: e92444. https://doi.org/10.1371/journal.pone.0092444

[27] Kim MH, Kim CG, Kim SK, Shin SJ, Choe EA et al. YAP-induced PD-L1 expression drives immune evasion in BRAFiresistant melanoma. Cancer Immunol Res 2018; 6: 255-266. https://doi.org/10.1158/2326-6066.CIR-17-0320

[28] Hanahan D, Weinberg RA. Hallmarks of cancer: the next generation. Cell 2011; 144: 646-674. https://doi.org/10.1016/j. cell.2011.02.013

[29] Green MR, Rodig S, Juszczynski P, Ouyang J, Sinha P et al. Constitutive AP-1 activity and EBV infection induce PD-L1 in Hodgkin lymphomasand posttransplant lymphoproliferative disorders: implications for targeted therapy. Clin Cancer Res 2012; 18: 1611-1618. https://doi.org/10.1158/1078-0432. CCR-11-1942 
[30] Liu J, Hamrouni A, Wolowiec D, Coiteaux V, Kuliczkowski $\mathrm{K}$ et al. Plasma cells from multiple myeloma patients express PD-L1 (PDL1) and increase expression after stimulation with IFN-\{gamma\} and TLR ligands via a MyD88-, TRAF6, and MEK-dependent pathway. Blood 2007; 110: 296-304. https://doi.org/10.1182/blood-2006-10-051482

[31] Qian Y, Deng J, Geng L, Xie H, Jiang G et al. TLR4 signaling induces PD-L1 expression through MAPK pathways in bladder cancer cells. Cancer Invest 2008; 26: 816-821. https://doi. org/10.1080/07357900801941852

[32] Shi J, Qin X, Zhao L, Wang G, Liu C. Human immunodeficiency virus type 1 Tat induces PD-L1 expression via ERK/ MAPK signaling pathway. Cell Immunol 2011; 271: 280-285. https://doi.org/10.1016/j.cellimm.2011.07.005

[33] Yamamoto R, Nishikori M, Tashima M, Sakai T, Ichinohe $\mathrm{T}$ et al. PD-L1 expression is regulated by MEK/ERK signaling pathway in anaplastic large cell lymphoma and Hodgkin lymphoma. Cancer Sci 2009; 100: 2093-2100. https://doi. org/10.1111/j.1349-7006.2009.01302.x

[34] Lee BS, Park DI, Lee DH, Lee JE, Yeo MK et al. Hippo effector YAP directly regulates the expression of PD-L1 transcripts in EGFR-TKI-resistant lung adenocarcinoma. Biochem Biophys Res Commun 2018; 497: 1184. https://doi. org/10.1016/j.bbrc.2018.03.006

[35] Monje P, Hernandez-Losa J, Lyons RJ, Castellone MD, Gutkind JS. Regulation of the transcriptional activity of c-Fos by ERK. A novel role for the prolyl isomerase PIN1. J Biol Chem 2005; 280: 35081-35084. https://doi.org/10.1074/jbc. C500353200

[36] Loi S, Dushyanthen S, Beavis PA, Salgado R, Denkert C et al. RAS/MAPK activation is associated with reduced tumor infiltrating lymphocytes in triple-negative breast cancer: therapeutic cooperation between MEK and PD-1/PD-L1 immune checkpoint inhibitors. Clin Cancer Res 2016; 22: 1499-1509. https://doi.org/10.1158/1078-0432.CCR-15-1125

[37] Audrito V, Serra S, Stingi A, Orso F, Gaudino F et al. PD-L1 up-regulation in melanoma increases disease aggressiveness and is mediated through miR-17-5p. Oncotarget 2017; 8: 15894-15911. https://doi.org/10.18632/oncotarget.15213

[38] Atefi M, Avramis E, Lassen A, Wong DJ, Robert L et al. Effects of MAPK and PI3K pathways on PD-L1 expression in melanoma. Clin Cancer Res 2014; 20: 3446-3457. https:// doi.org/10.1158/1078-0432.CCR-13-2797
[39] Prahallad A, Sun C, Huang S, Di Nicolantonio F, Salazar R et al. Unresponsiveness of colon cancer to BRAF (V600E) inhibition through feedback activation of EGFR. Nature 2012; 483: 100-103. https://doi.org/10.1038/nature10868

[40] Corcoran RB, Andre T, Atreya CE, Schellens JHM, Yoshino $\mathrm{T}$ et al. Combined BRAF, EGFR, and MEK inhibition in patients with BRAF(V600E)-mutant colorectal cancer. Cancer Discov 2018; 8: 428-443. https://doi.org/10.1158/2159-8290. CD-17-1226

[41] Liu J, Quan L, Zhang C, Liu A, Tong D et al. Over-activated PD-1/PD-L1 axis facilitates the chemoresistance of diffuse large B-cell lymphoma cells to the CHOP regimen. Oncol Lett 2018; 15: 3321-3328. https://doi.org/10.3892/ ol.2017.7682

[42] Yan F, Pang J, Peng Y, Molina JR, Yang P et al. Elevated cellular PD1/PD-L1 expression confers acquired resistance to cisplatin in small cell lung cancer cells. PLoS One 2016; 11: e0162925. https://doi.org/10.1371/journal.pone.0162925

[43] Zhang XH, Tee LY, Wang XG, Huang QS, Yang SH. Off-target effects in CRISPR/Cas9-mediated genome engineering. Mol Ther Nucleic Acids 2015; 4: e264. https://doi.org/10.1038/ mtna.2015.37

[44] Schluter C, Duchrow M, Wohlenberg C, Becker MH, Key $\mathrm{G}$ et al. The cell proliferation-associated antigen of antibody Ki-67: a very large, ubiquitous nuclear protein with numerous repeated elements, representing a new kind of cell cycle maintaining proteins. J Cell Biol 1993; 123: 513-522. https:// doi.org/10.1083/jcb.123.3.513

[45] Mebratu Y, Tesfaigzi Y. How ERK1/2 activation controls cell proliferation and cell death: Is subcellular localization the answer? Cell Cycle 2009; 8: 1168-1175. https://doi. org/10.4161/cc.8.8.8147

[46] Chang CH, Qiu J, O'Sullivan D, Buck MD, Noguchi T et al. Metabolic Competition in the Tumor Microenvironment Is a Driver of Cancer Progression. Cell 2015; 162: 1229-1241. https://doi.org/10.1016/j.cell.2015.08.016

[47] Phong MS, Van Horn RD, Li S, Tucker-Kellogg G, Surana $\mathrm{U}$ et al. p38 mitogenactivated protein kinase promotes cell survival in response to DNA damage but is not required for the G(2) DNA damage checkpoint in human cancer cells. Mol Cell Biol 2010; 30: 3816-3826. https://doi.org/10.1128/ MCB.00949-09

[48] Adams JM, Cory S. The BCL-2 arbiters of apoptosis and their growing role as cancer targets. Cell Death Differ 2018; 25 : 27-36. https://doi.org/10.1038/cdd.2017.161 